\title{
FGF21 as an Endocrine Regulator in Lipid Metabolism: From Molecular Evolution to Physiology and Pathophysiology
}

\author{
Yusuke Murata, Morichika Konishi, and Nobuyuki Itoh \\ Department of Genetic Biochemistry, Kyoto University Graduate School of Pharmaceutical Sciences, Sakyo, Kyoto 606-8501, Japan \\ Correspondence should be addressed to Nobuyuki Itoh, itohnobu@pharm.kyoto-u.ac.jp \\ Received 14 November 2010; Accepted 5 January 2011 \\ Academic Editor: Chandan Prasad \\ Copyright () 2011 Yusuke Murata et al. This is an open access article distributed under the Creative Commons Attribution License, \\ which permits unrestricted use, distribution, and reproduction in any medium, provided the original work is properly cited.
}

The FGF family comprises twenty-two structurally related proteins with functions in development and metabolism. The Fgf21 gene was generated early in vertebrate evolution. FGF21 acts as an endocrine regulator in lipid metabolism. Hepatic Fgf21 expression is markedly induced in mice by fasting or a ketogenic diet. Experiments with Fgf21 transgenic mice and cultured cells indicate that FGF21 exerts pharmacological effects on glucose and lipid metabolism in hepatocytes and adipocytes via cell surface FGF receptors. However, experiments with Fgf21 knockout mice indicate that FGF21 inhibits lipolysis in adipocytes during fasting and attenuates torpor induced by a ketogenic diet but maybe not a physiological regulator for these hepatic functions. These findings suggest the pharmacological effects to be distinct from the physiological roles. Serum FGF21 levels are increased in patients with metabolic diseases having insulin resistance, indicating that FGF21 is a metabolic regulator and a biomarker for these diseases.

\section{Background}

Prototypes of fibroblast growth factors (FGFs), FGF1 and FGF2, were originally isolated as mitogens for cultured fibroblasts from the brain and pituitary $[1,2]$. The human/ mouse Fgf gene family comprises twenty-two members, including $F g f 1-F g f 23$, all of which are evolutionarily related. However, as mouse Fgf15 and human Fgf19 are orthologous, we refer to these genes as $F g f 15 / 19$ in this paper. Human/mouse FGFs are proteins of $\sim 150-300$ amino acids with $13-71 \%$ identity. All FGFs with a $\sim 120$ amino acid conserved core region $(\sim 30-60 \%$ identity) are signaling molecules with diverse functions in development and metabolism. Fgf genes are widely expressed in developing and adult tissues [3-6].

FGFs can be classified into three types, paracrine, intracrine, and endocrine FGFs, by their mechanisms of action [6]. Paracrine FGFs (FGF1 FGF10, FGF16 FGF18, FGF20, and FGF22) function as secreted local paracrine signaling molecules in multiple developmental processes, including differentiation, cell proliferation, and migration. They mediate biological responses by binding to cell surface tyrosine kinase FGF receptors (FGFRs) $[4,6,7]$. Intracrine
FGFs (FGF11 FGF14) function as nonsecreted signaling molecules. They mainly play roles in neuronal functions at postnatal stages in an FGFR-independent manner [8-10]. Endocrine FGFs (FGF15/19, FGF21, and FGF23) mediate biological responses as secreted proteins in an FGFRdependent manner. Endocrine FGFs function over long distances in an endocrine manner and mainly play roles in metabolism at postnatal stages [11-13].

Paracrine and intracrine FGFs have been identified in both invertebrates and vertebrates. However, endocrine FGFs have only been identified in vertebrates [6]. Endocrine FGFs are emerging in evolution. FGF15/19 and FGF23 play roles as metabolic regulators in bile acid metabolism and phosphate and active vitamin D metabolism, respectively [11, 13-15]. FGF21 exerts diverse pharmacological effects on glucose and lipid metabolism, ketogenesis, and growth hormone signaling in hepatocytes in mice. However, FGF21 may not be a physiological regulator for these hepatic functions. FGF21 physiologically regulates lipid metabolism in adipocytes and torpor. Serum FGF21 levels are significantly increased in patients with metabolic diseases having insulin resistance. These results indicate the physiological and pathophysiological roles of FGF21. Several excellent review articles on FGF21, 
focusing on its pharmacological effects on metabolism and therapeutic uses for metabolic diseases have been published [16-20]. This paper concentrates on the molecular evolution and physiological and pathophysiological roles of FGF21.

\section{Identification of Fgf21}

The Fgf21 gene was originally identified in mice by the polymerase chain reaction with the amino acid sequence of human FGF15/19. Human Fgf21 was also identified by homology-based searching in the human DNA database [21]. Later, human FGF21 was found to act as a stimulator of glucose uptake in mouse 3T3-L1 adipocytes in an assay used to search for novel proteins with therapeutic potential to treat diabetes [22]. Mouse Fgf21 was also identified as a hepatic gene inducible by fasting or a ketogenic diet $[23,24]$.

Human FGF21 is a secreted protein of 209 amino acids with a 29 -amino-acid amino-terminal secreted signal sequence and a $\sim 120$-amino-acid conserved core region. Human FGF21 is highly homologous to mouse FGF21 ( $\sim 75 \%$ identity). However, its low homology with other human FGFs (less than 35\% identity) indicates that FGF21 is structurally unique [21]. Paracrine FGFs have a heparinbinding site, which is necessary for stable interaction with FGFRs and heparin-like molecules [25]. However, FGF21 as well as FGF15/19 and FGF23 lack a typical heparin-binding site $[25,26]$.

\section{Molecular Evolution of Fgf21}

The FGF signaling system has been conserved throughout metazoan evolution. Potential evolutionary relationships in the Fgf family have been proposed based on results of gene location and phylogenetic analyses. These analyses have identified seven subfamilies: $\mathrm{Fgf} / 1 / 2 / 5, \mathrm{Fgf3} / 4 / 6, \mathrm{Fgf7} / 10 / 22$, Fgf8/17/18, Fgf9/16/20, Fgf11/12/13/14, and Fgf15/19/21/23 $[4,6]$. Ascidians belong to the subphylum Urochordata, the earliest branch in the phylum Chordata. Ancestral genes of paracrine and intracrine $F g f s$ have been identified in the ascidian, Ciona intestinalis [27]. However, no ancestral gene of endocrine $F g f_{s}$ has been identified in Ciona intestinalis. The sea lampreys, Petromyzon marinus, are cyclostomes, the most basal extant group of vertebrates [28]. An ancestral gene of endocrine $\mathrm{Fg} f \mathrm{~s}$ has been identified in the lamprey genome, and tentatively named Fgf15/19like (Itoh et al., unpublished observation) (Figure 1(a)). Lamprey FGF15/19-like also lacks a typical heparin-binding site. These results support that lamprey Fgf15/19-like is an ancestral endocrine $F g$, which was generated from the ancestral paracrine Fgf gene, Fgf4-like, by local gene duplication early in vertebrate evolution (Figure 1(b)). Later, $F g f 19, F g f 21$, and $F g f 23$ were generated from the ancestral endocrine $\mathrm{Fg} f$ gene by two genome duplication events early in vertebrate evolution $[4,6]$. The evolutionary history suggests that endocrine FGFs are vertebrate specific. As described above, paracrine FGFs have a heparin-binding site. The site is necessary for the stable binding of FGFRs/heparinlike molecules and local signaling in a paracrine manner.
Endocrine FGFs potentially acquired systemic signaling in an endocrine manner by reducing heparin-binding affinity early in vertebrate evolution $[6,13,25]$.

Endocrine $F g f$ genes have been identified in all vertebrates examined, including teleosts, amphibians, reptiles, birds, and mammals. Fgf21 has also been identified in most vertebrates. However, $F g f 21$ has not been identified in the chicken and zebra finch genomes (Ensemble Genome Browser; Itoh et al., unpublished observation) (Figure 1(a)). Genome sizes and gene numbers are smaller in birds than in mammalian species. Although the evolutionary implications of these changes remain to be understood, the reduced genome sizes and gene numbers may have evolved in response to the physiological demands of flight [29]. Fgf21 might therefore have been lost in the bird lineage.

\section{Roles of FGF21 in Glucose Metabolism}

Fgf21 is expressed abundantly in the liver, and also in the pancreas, white adipose tissue, muscle, and testis $[17,21,30]$. Potential roles of FGF21 in glucose metabolism were first shown by experiments with cultured cells [22]. FGF21 stimulated glucose uptake in cultured mouse and human adipocytes. Functional interplay between the FGF21 and peroxisome proliferation-activated receptor $\gamma(\operatorname{PPAR} \gamma)$ pathways led to a marked stimulation of glucose transport, suggesting a novel synergy between FGF21 and PPAR $\gamma$ homeostasis [31]. In addition, Fgf21 transgenic mice were resistant to diet-induced obesity. Serum glucose levels were also reduced to near normal levels in both $o b / o b$ and $d b / d b$ mice by the administration of FGF21. These findings indicate that FGF21 plays a role in glucose metabolism and has potential therapeutic effects on metabolic diseases [16-19].

To elucidate the physiological roles of FGF21, Fgf21 knockout mice have been generated. These mice had normal food intake and energy expenditure levels, serum glucose and insulin levels, and hepatic glycogen levels, indicating that FGF21 is not to be a physiological regulator for glucose metabolism [32].

\section{Roles of FGF21 in Lipolysis in Adipocytes}

Mammals have evolved complex metabolic responses to fasting. During fasting, nonesterified fatty acid (NEFA) is released from adipocytes into the blood and taken up by hepatocytes. Peroxisome proliferator-activated receptor $\alpha$ (PPAR $\alpha$ ) is a nuclear receptor. Hepatic Fgf21 expression was greatly induced by fasting for $24 \mathrm{~h}$ in wild-type mice but not PPAR $\alpha$ knockout mice. It was also markedly induced by a PPAR $\alpha$-selective agonist [23]. These results indicate that hepatic Fgf21 expression is induced by the activation of PPAR $\alpha$. NEFA binds to and activates PPAR $\alpha$. The ligandbound PPAR $\alpha$ forms a heterodimer with RXRs and induces the expression of $F g f 21$ [33]. Fasting increases the amount of NEFA released from adipocytes. Hepatic Fgf21 expression during fasting is probably induced through the activation of PPAR $\alpha$ by NEFA (Figure 2(a)) [34, 35]. Fgf21 knockout mice fasted for $24 \mathrm{~h}$ showed increased lipolysis in adipocytes, 


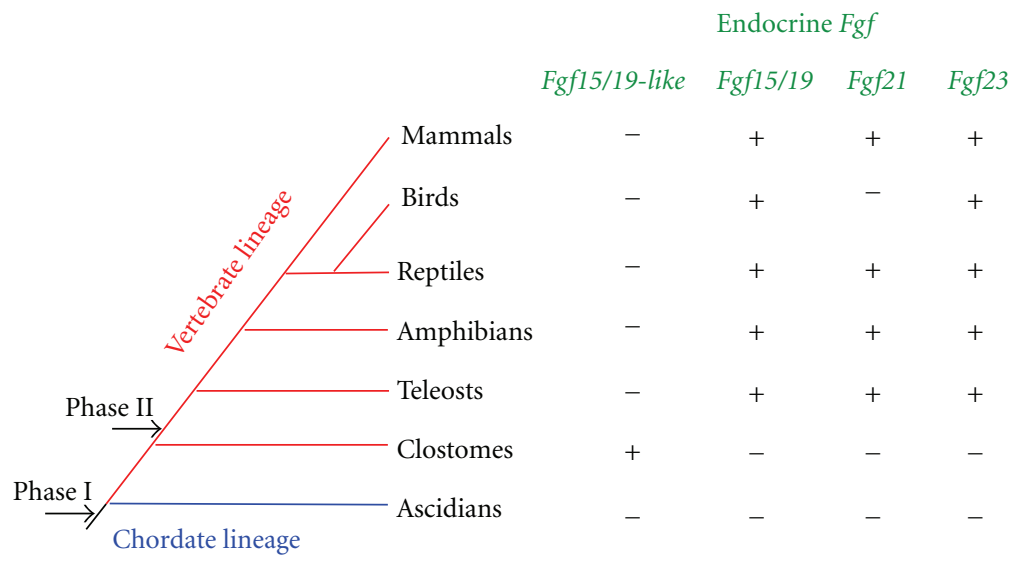

Deuterostome lineage

(a)

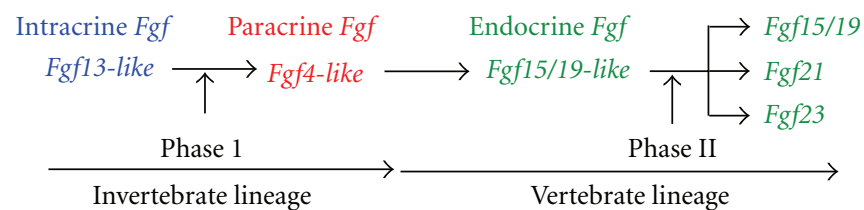

(b)

FIguRe 1: The evolutionary lineage of deuterostome organisms and the evolutionary history of endocrine Fgf genes. (a) The Fgf gene family expanded in two major phases (I and II) during deuterostome evolution. Phase I occurred after the separation of protostomes and deuterostomes. Phase II occurred early in the emergence of vertebrates. (b) Fgf13-like is the ancestral gene of the Fgf gene family. Fgf4-like was generated from Fgf13-like by gene duplication during invertebrate evolution. Fgf15/19-like was also generated from Fgf4-like by local gene duplication early in the emergence of vertebrates. Later, Fgf19, Fgf21, and Fgf23 were generated via two genome duplication events in phase II.

which resulted in decreased adipocyte size and increased serum NEFA levels [32]. These results indicate that FGF21 inhibits lipolysis in adipocytes during fasting. The regulatory process forms a negative feedback loop in the control of lipolysis by FGF21 (Figure 2(a)). FGF21 also regulates mitochondrial activity and enhances oxidative capacity through an AMP-activated protein kinase- (AMPK-) sirtuin 1- (SIRT1-) peroxisome proliferator-activated receptor- $\gamma$ coactivator- $1 \alpha$ (PGC-1 $\alpha$-) dependent mechanism in adipocytes [36].

\section{Roles of FGF21 in Ketogenesis and Triglyceride Clearance in Hepatocytes}

In hepatocytes, NEFA is converted to acetyl-CoA by oxidation, and ketone bodies are produced from acetyl-CoA. Ketone bodies become the predominant energy source for the brain during fasting. Hepatic ketogenesis during fasting was greatly impaired in $P P A R \alpha$ knockout mice, indicating that $\operatorname{PPAR} \alpha$ is crucial to the normal adaptive response to fasting [37, 38]. As described above, hepatic Fgf21 expression is induced in response to fasting and PPAR $\alpha$ agonists. In addition, the phenotypes of Fgf21 transgenic mice demonstrate that FGF21 stimulates hepatic ketogenesis, indicating that FGF21 plays a role in hepatic ketogenesis [23]. Feeding with a ketogenic diet (KD) mimics the metabolic conditions of chronic starvation. Adenoviral knockdown of hepatic Fgf21 in mice fed KD caused reduced blood ketone levels, fatty liver, and lipemia, suggesting that FGF21 is required for hepatic ketogenesis and triglyceride clearance in mice fed KD [24]. In addition, serum triglyceride levels were reduced to near normal levels in both $o b / o b$ and $d b / d b$ mice by the administration of FGF21 [22]. These findings also indicate functions of FGF21 in ketogenesis and triglyceride metabolism and potential therapeutic effects on metabolic diseases [16-19]. However, hepatic ketogenesis and triglyceride levels were essentially normal in Fgf21 knockout mice fasted or fed KD, indicating FGF21 not to be a physiological regulator for hepatic ketogenesis and triglyceride clearance in mice [32]. These results suggest the physiological roles of FGF21 to be distinct from the pharmacological effects of FGF21 indicated by experiments with Fgf21 transgenic mice. In humans, serum FGF21 levels are also increased by fasting for 7 days or PPAR $\alpha$ activation. In contrast, ketogenesis is independent of serum FGF21 levels [39, 40]. However, it also has been reported that Fgf21 knockout mice fed KD developed hepatosteatosis and showed partial impairment in ketogenesis [41].

Peroxisome proliferation-activated receptor $\gamma$ coactivator- $1 \alpha(\mathrm{PGC}-1 \alpha)$ regulates metabolism in response to changes in nutritional status. PGC- $1 \alpha$ negatively regulated hepatic Fgf21 expression [42]. In contrast, FGF21 induced hepatic Pgc-1 $\alpha$ expression. Fgf21 knockout mice did not fully express $P g c-1 \alpha$ in response to prolonged fasting and exhibited 


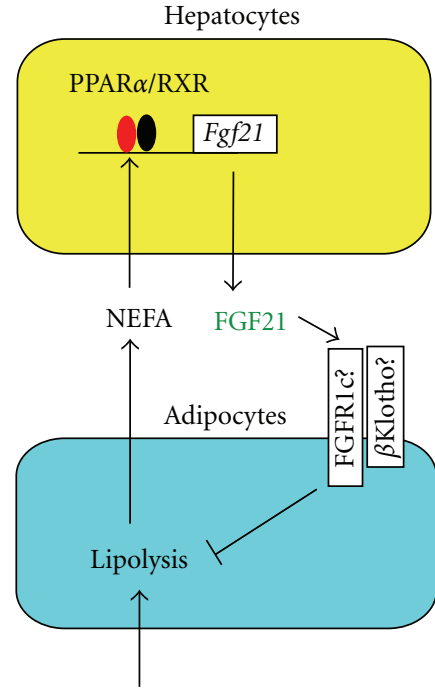

Starvation

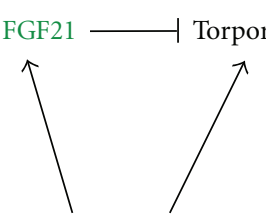

Ketogenic diet (b)

Figure 2: Mechanism of action and regulatory mechanism of the gene expression of FGF21. (a) Hepatic Fgf21 expression is induced by the activation of PPAR $\alpha$. NEFA binds to and activates PPAR $\alpha$. The ligand-bound PPAR $\alpha$ forms a heterodimer with RXRs and induces the expression of Fgf21. The FGF21 inhibits lipolysis in adipocytes. In cultured adipocytes, FGF21 signaling is transduced by activating the $\beta$ Klotho-FGFR1c complex. However, FGF2 1 signaling can be transduced in $\beta$ Klotho knockout mice, indicating the existence of a $\beta$ Klotho-independent FGF21 signaling pathway. The regulatory process forms a negative feedback loop in the control of lipolysis by FGF21. (b) Feeding with a ketogenic diet (KD) mimics the metabolic conditions of chronic starvation. KD induces hepatic Fgf21 expression and torpor. FGF21 attenuates torpor induced by KD.

impaired gluconeogenesis and ketogenesis. In addition, FGF21 could not induce gluconeogenic gene expression in $P g c-1 \alpha$ knockout mice [43]. These results indicate that gluconeogenesis and ketogenesis by FGF21 are mediated in part through PGC-1 $\alpha$. However, as described above, other experiments with Fgf21 knockout mice suggest that FGF21 may not be required for gluconeogenesis and ketogenesis [32].

\section{Roles of FGF21 in Growth Hormone Signaling in Hepatocytes}

Starvation inhibits growth by blocking the growth hormone (GH)/insulin-like growth factor 1 (IGF1) signaling pathway [44]. Fgf21 transgenic mice are 40-50\% smaller than their wild-type counterparts. Tibia length is also significantly reduced in Fgf21 transgenic mice. FGF21 causes resistance to $\mathrm{GH}$ in the liver [45]. Actions of $\mathrm{GH}$ are mostly mediated by IGF1. Igf1 expression is induced by the GH/STAT5 (signal transducer and activator of transcription 5) signaling pathway. The phosphorylation of STAT5 and the expression of Igf1 are significantly decreased in livers of $F g f 21$ transgenic mice. IGF-binding protein 1 (IGFBP1), which is involved

in sequestering IGF1, inhibits IGF1 signaling. A suppressor of cytokine signaling 2 (SOCS2) also inhibits GH signaling by binding to the tyrosine-phosphorylated $\mathrm{GH}$ receptor. The expression of Igfbp1 and Socs 2 was greatly enhanced in Fgf21 transgenic livers. These results indicate the important role of FGF21 in the inhibition of GH/IGF1 signaling [45]. However, Fgf21 knockout mice are apparently healthy, and their body and tibia lengths are essentially normal [32]. In addition, hepatic $I g f 1, I g f b p 1$, and Socs 2 expression was essentially normal in Fgf21 knockout mice (Murata et al., unpublished observation). The expression of Igf1 was slightly decreased by fasting for $24 \mathrm{~h}$ in wild-type mice. In contrast, the expression of $I g f b p 1$ and Socs 2 was greatly and slightly increased in fasted wild-type mice, respectively. In addition, the expression of $I g f 1, I g f b p 1$, and Socs 2 in fasted Fgf21 knockout mice was similar to that in fasted wildtype mice (Murata et al., unpublished observation). The Fgf21 knockout phenotypes indicate that FGF21 is not a physiological regulator essential for GH/IGF1 signaling.

\section{Roles of FGF21 in Torpor}

Torpor, the controlled lowering of metabolic rates, body temperature, and physical activity, is an adaptation that various mammals use to cope with periods of low food availability [46]. The basal core body temperature of Fgf21 transgenic mice is consistently lower than that of wildtype mice. Moreover, Fgf21 transgenic mice enter torpor on fasting for $24 \mathrm{~h}$, whereas wild-type mice do not [23]. In addition, a PPAR pan-agonist reduced body temperature late at night in concert with the induction of hepatic of Fgf21 expression [47]. However, body temperature and physical activity were essentially normal in Fgf21 knockout mice fasted for $24 \mathrm{~h}$, indicating that FGF21 is not physiologically

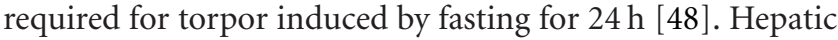
$\mathrm{Fgf} 21$ expression and torpor were also induced in mice fed KD for 5 days (Figure 2(b)). However, torpor was attenuated in Fgf21 knockout mice fed KD for 5 days. These results indicate that FGF21 is potentially involved in the torpor induced by KD [48] (Figure 2(b)).

\section{Mechanism of FGF21 Action}

FGF signaling is mostly mediated by the activation of FGFRs. Four Fgfr genes, Fgfr1-Fgfr4, have been identified in humans and mice $[3,7,49]$. These genes encode proteins $(\sim 800$ amino acids) that contain an extracellular ligand-binding domain with three immunoglobulin-like domains (I, II, and III), a transmembrane domain, and intracellular tyrosine kinase domains. Fgfr1-Fgfr3 encode two major variants of immunoglobin-like domain III (IIIb and IIIc) generated by alternative splicing. The immunoglobulin-like domain III is an essential determinant of ligand-binding specificity [26]. Thus, seven FGFR proteins (FGFR 1b, 1c, 2b, 2c, 3b, 3c, and 4) with differing ligand-binding specificity are generated from Fgfr1-Fgfr4. The binding of FGFs to FGFRs induces receptor dimerization and transphosphorylation and the 
activation of downstream signaling pathways: RAS-RAFMAPK, PI3K-AKT, STAT, and PLC $\gamma$ [7, 49].

Endocrine FGFs also mediate biological responses in an FGFR-dependent manner. However, they activate FGFRs with very low activity even in the presence of heparin/heparan sulfate, as they bind to heparin/heparan sulfate with very low affinity $[25,26] . \alpha$ Klotho is a transmembrane protein of $\sim 1,000$ amino acids with a short cytoplasmic domain [50]. $\beta$ Klotho shares structural identity ( $41 \%$ amino acid identity) and characteristics with $\alpha$ Klotho [51]. Fgf23 and $\alpha$ Klotho knockout mouse phenotypes indicate that FGF23 and $\alpha$ Klotho function in a common signal transduction pathway. FGF23 can bind to the $\alpha$ Klotho-FGFR1c complex in cultured cells [52], suggesting that $\alpha$ Klotho is a cofactor essential for FGF23 signaling. $\beta$ Klotho, Fgf15/19, and Fgfr4 knockout mouse phenotypes also indicate that FGF15/19, FGFR4, and $\beta$ Klotho function in a common signal transduction pathway [53-55]. FGF15/19 can bind to the $\beta$ Klotho-FGFR 4 complex in cultured cells. FGF15/19 also activates FGF signaling in hepatocytes that primarily express Fgfr4 [56].

In the presence of $\beta$ Klotho, FGF21 can bind to and activate FGFR1c, FGFR2c, FGFR3c, or FGFR4, which activates the MAP kinase pathway, in cultured cells, indicating that $\beta$ Klotho is also essential for FGF21 signaling in cultured cells [57-59]. However, Fgf21 knockout mouse phenotypes [32] are distinct from $\beta$ Klotho knockout mouse phenotypes [51]. In addition, the administration of a recombinant human FGF21 to $\beta$ Klotho knockout mice showed that FGF21 signaling is transduced in the absence of $\beta$ Klotho [60]. These results indicate the existence of a $\beta$ Klothoindependent FGF21 signaling pathway in which undefined cofactors might be involved [60].

\section{FGF21 Signaling in Metabolic Diseases}

Nonalcoholic fatty liver disease (NAFLD) is a hepatic manifestation of metabolic syndrome and ranges from simple fatty liver to nonalcoholic steatohepatitis. Its prevalence has increased dramatically over recent years in developed countries [61]. The pathophysiological hallmark of NAFLD is insulin resistance. NAFLD may increase the risk of type 2 diabetes and atherosclerosis [62]. Serum FGF21 levels are significantly increased in NAFLD (Table 1) [63-65]. Serum FGF21 levels are positively correlated with intrahepatic triglyceride levels [65]. As NAFLD is now recognized as a major public health problem, reliable biomarkers for NEFLD are needed. Serum FGF21 levels might be useful as a biomarker for NEFLD [61].

Type 2 diabetes connected with visceral obesity and insulin resistance has become a global health concern. Serum FGF21 levels are increased in patients with type 2 diabetes, gestational diabetes, and obesity, indicating FGF21 to be a potential new marker in patients with type 2 diabetes (Table 1) [66-71]. Serum FGF21 levels are independently associated with markers of insulin resistance and an adverse lipid profile $[66,70]$. The upregulation of serum FGF21 levels might be a compensatory mechanism to improve
TABLE 1: Increased serum FGF21 levels in metabolic disease patients with insulin resistance.

\begin{tabular}{llc}
\hline Disease & $\begin{array}{c}\text { Insulin } \\
\text { signaling }\end{array}$ & $\begin{array}{c}\text { Serum FGF21 } \\
\text { levels }\end{array}$ \\
\hline Nonalcoholic fatty liver disease & Resistance & Increase \\
Type 2 diabetes & Resistance & Increase \\
Obesity & Resistance & Increase \\
Cushing's syndrome & Resistance & Increase \\
Lipodystrophy induced by HIV-1 & Resistance & Increase \\
Endostage renal disease & Resistance & Increase \\
\hline
\end{tabular}

glucose metabolism when insulin resistance is present. Dietinduced obese mice also have increased serum (endogenous) FGF21 levels and respond poorly to exogenous FGF21, indicating that obesity is an FGF21-resistant state [72]. Impaired glucose tolerance (IGT) is an important category of prediabetes. Serum FGF21 levels are also increased in Chinese subjects with IGT. However, serum FGF21 levels do not correlate with insulin resistance in the subjects [73].

Cushing's syndrome is a hormone disorder caused by high levels of cortisol (hypercortisolism) in the blood. Patients with Cushing's syndrome frequently suffer from visceral obesity, insulin resistance/diabetes, and other abnormalities similarly to patients with metabolic syndrome. Serum FGF21 levels are also increased in patients with Cushing's syndrome. The increased FGF21 levels are due to excessive fat accumulation and related metabolic abnormalities rather than a direct effect of cortical on FGF21 production (Table 1) [74].

Lipodystrophy is a common alteration in HIV-1-infected patients under antiretroviral treatment. This syndrome is usually associated with peripheral lipoatrophy, central adiposity, and, in some cases, lipomatosis, as well as systemic insulin resistance and hyperlipidemia [75]. Serum FGF21 levels are increased in HIV-1-infected patients with lipodystrophy. This increase is closely associated with insulin resistance, metabolic syndrome, and markers of liver damage. FGF21 might be a biomarker of altered metabolism in HIV1-infected, antiretroviral-treated patients (Table 1) [76].

Serum FGF21 levels correlate with renal function and are markedly increased in chronic kidney disease patients receiving hemodialysis, suggesting a possible link between their FGF21 levels and renal function [77]. Patients with endstage renal disease (ESRD) show insulin resistance. Serum FGF21 levels are also markedly increased in patients with ESRD, suggesting FGF21 to play a role in insulin resistance in these patients (Table 1) [78].

\section{Conclusion}

Endocrine FGFs, FGF15/19, FGF21, and FGF23, are emerging in evolution and unique in function. The Fgf21 gene, which was generated early in vertebrate evolution, is specific to vertebrates. $F g f 21$ has been identified in most vertebrate genomes, but not in bird genomes, indicating that it might be lost in the bird lineage. Genome sizes and gene numbers 
are smaller in birds than in mammalian species. As the differences might have evolved in response to the physiological demands of flight, Fgf21 also might have been lost. FGF21 mainly acts as an endocrine factor in an FGFR-dependent manner. FGF21 requires $\beta$ Klotho as a cofactor in cultured cells. However, it may not require $\beta$ Klotho in mice. FGF2 1 exerts pharmacological effects on hepatic glucose and lipid metabolism and growth hormone signaling. These effects might be useful for treating metabolic diseases. However, experiments with Fgf21 knockout mice indicated FGF21 not to be physiologically essential for hepatic glucose and lipid metabolism, ketogenesis, and growth hormone signaling. In contrast, FGF21 inhibited lipolysis in adipocytes of fasted mice and attenuated torpor induced by KD, indicating that Fgf21 may be a "thrifty gene." Serum FGF21 levels are increased in patients with metabolic diseases having insulin resistance including NAFLD, type 2 diabetes, Cushing's syndrome, and HIV-1-induced lipodystrophy. Although it remains unclear whether serum FGF21 levels are increased by FGF21 resistance or an adaptive response to metabolic disorders, these findings indicate that FGF21 potentially functions as a metabolic regulator in relation with insulin resistance and is a biomarker for metabolic diseases. Further study of FGF21 may provide clues as to its roles in lipid metabolism and clinical treatments for metabolic diseases.

\section{Acknowledgment}

This work was supported by a Grant-in-aid for Scientific Research from the Ministry of Education, Science, Culture and Sports of Japan and the Takeda Science Foundation, Japan.

\section{References}

[1] D. Gospodarowicz, "Purification of a fibroblast growth factor from bovine pituitary," Journal of Biological Chemistry, vol. 250, no. 7, pp. 2515-2520, 1975.

[2] D. Gospodarowicz, H. Bialecki, and G. Greenburg, "Purification of the fibroblast growth factor activity from bovine brain," Journal of Biological Chemistry, vol. 253, no. 10, pp. 37363743, 1978.

[3] N. Itoh and D. M. Ornitz, "Evolution of the Fgf and Fgfr gene families," Trends in Genetics, vol. 20, no. 11, pp. 563-569, 2004.

[4] N. Itoh and D. M. Ornitz, "Functional evolutionary history of the mouse Fgf gene family," Developmental Dynamics, vol. 237, no. 1, pp. 18-27, 2008.

[5] N. Itoh, "The Fgf families in humans, mice, and zebrafish: their evolutional processes and roles in development, metabolism, and disease," Biological and Pharmaceutical Bulletin, vol. 30, no. 10, pp. 1819-1825, 2007.

[6] N. Itoh and D. M. Ornitz, "Fibroblast growth factors: from molecular evolution to roles in development, metabolism and disease," Journal of Biochemistry, vol. 149, no. 2, pp. 121-130, 2011.

[7] A. Beenken and M. Mohammadi, "The FGF family: biology, pathophysiology and therapy," Nature Reviews Drug Discovery, vol. 8, no. 3, pp. 235-253, 2009.
[8] M. Goldfarb, "Fibroblast growth factor homologous factors: evolution, structure, and function," Cytokine and Growth Factor Reviews, vol. 16, no. 2, pp. 215-220, 2005.

[9] M. Goldfarb, J. Schoorlemmer, A. Williams et al., "Fibroblast growth factor homologous factors control neuronal excitability through modulation of voltage-gated sodium channels," Neuron, vol. 55, no. 3, pp. 449-463, 2007.

[10] M. Xiao, L. Xu, F. Laezza, K. Yamada, S. Feng, and D. M. Ornitz, "Impaired hippocampal synaptic transmission and plasticity in mice lacking fibroblast growth factor 14," Molecular and Cellular Neuroscience, vol. 34, no. 3, pp. 366377, 2007.

[11] H. Kurosu and M. Kuro-O, "Endocrine fibroblast growth factors as regulators of metabolic homeostasis," BioFactors, vol. 35, no. 1, pp. 52-60, 2009.

[12] A. Kharitonenkov, "FGFs and metabolism," Current Opinion in Pharmacology, vol. 9, no. 6, pp. 805-810, 2009.

[13] N. Itoh, "Hormone-like (endocrine) Fgfs: their evolutionary history and roles in development, metabolism, and disease," Cell and Tissue Research, vol. 342, no. 1, pp. 1-11, 2010.

[14] A. Kharitonenkov, "FGFs and metabolism," Current Opinion in Pharmacology, vol. 9, no. 6, pp. 805-810, 2009.

[15] M. S. Razzaque, "The FGF23-Klotho axis: endocrine regulation of phosphate homeostasis," Nature Reviews Endocrinology, vol. 5, no. 11, pp. 611-619, 2009.

[16] A. Kharitonenkov and A. B. Shanafelt, "Fibroblast growth factor-21 as a therapeutic agent for metabolic diseases," BioDrugs, vol. 22, no. 1, pp. 37-44, 2008.

[17] A. Kharitonenkov and A. B. Shanafelt, "FGF21: a novel prospect for the treatment of metabolic diseases," Current Opinion in Investigational Drugs, vol. 10, no. 4, pp. 359-364, 2009.

[18] S. A. Kliewer and D. J. Mangelsdorf, "Fibroblast growth factor 21: from pharmacology to physiology," American Journal of Clinical Nutrition, vol. 91, no. 1, pp. 254S-257S, 2010.

[19] D. Cuevas-Ramos, P. Almeda-Valdes, C. A. Aguilar-Salinas, G. Cuevas-Ramos, A. A. Cuevas-Sosa, and F. J. Gomez-Perez, "The role of fibroblast growth factor 21 (FGF21) on energy balance, glucose and lipid metabolism," Current Diabetes Reviews, vol. 5, no. 4, pp. 216-220, 2009.

[20] M. Rydén, "Fibroblast growth factor 21: an overview from a clinical perspective," Cellular and Molecular Life Sciences, vol. 66, no. 13, pp. 2067-2073, 2009.

[21] T. Nishimura, Y. Nakatake, M. Konishi, and N. Itoh, "Identification of a novel FGF, FGF-21, preferentially expressed in the liver," Biochimica et Biophysica Acta, vol. 1492, no. 1, pp. 203206, 2000.

[22] A. Kharitonenkov, T. L. Shiyanova, A. Koester et al., "FGF-21 as a novel metabolic regulator," Journal of Clinical Investigation, vol. 115, no. 6, pp. 1627-1635, 2005.

[23] T. Inagaki, P. Dutchak, G. Zhao et al., "Endocrine regulation of the fasting response by PPARalpha-mediated induction of fibroblast growth factor 21," Cell Metabolism, vol. 5, no. 6, pp. 415-425, 2007.

[24] M. K. Badman, P. Pissios, A. R. Kennedy, G. Koukos, J. S. Flier, and E. Maratos-Flier, "Hepatic fibroblast growth factor 21 is regulated by PPARalpha and is a key mediator of hepatic lipid metabolism in ketotic states," Cell Metabolism, vol. 5, no. 6, pp. 426-437, 2007.

[25] R. Goetz, A. Beenken, O. A. Ibrahimi et al., "Molecular insights into the Klotho-dependent, endocrine mode of action of fibroblast growth factor 19 subfamily members," Molecular and Cellular Biology, vol. 27, no. 9, pp. 3417-3428, 2007. 
[26] X. Zhang, O. A. Ibrahimi, S. K. Olsen, H. Umemori, M. Mohammadi, and D. M. Ornitz, "Receptor specificity of the fibroblast growth factor family: the complete mammalian FGF family," Journal of Biological Chemistry, vol. 281, no. 23, pp. 15694-15700, 2006.

[27] Y. Satou, K. S. Imai, and N. Satoh, "Fgf genes in the basal chordate Ciona intestinalis," Development Genes and Evolution, vol. 212, no. 9, pp. 432-438, 2002.

[28] N. Nikitina, M. Bronner-Fraser, and T. Sauka-Spengler, "The sea lamprey Petromyzon marinus: a model for evolutionary and developmental biology," Cold Spring Harbor Protocols, vol. 1, pp. 405-429, 2009.

[29] H. Ellegren, "The avian genome uncovered," Trends in Ecology and Evolution, vol. 20, no. 4, pp. 180-186, 2005.

[30] K. F. Tacer, A. L. Bookout, X. Ding et al., "Research resource: comprehensive expression atlas of the fibroblast growth factor system in adult mouse," Molecular Endocrinology, vol. 24, no. 10, pp. 2050-2064, 2010.

[31] J. S. Moyers, T. L. Shiyanova, F. Mehrbod et al., "Molecular determinants of FGF-21 activity-synergy and cross-talk with PPAR $y$ signaling," Journal of Cellular Physiology, vol. 210, no. 1, pp. 1-6, 2007.

[32] Y. Hotta, H. Nakamura, M. Konishi et al., "Fibroblast growth factor 21 regulates lipolysis in white adipose tissue but is not required for ketogenesis and triglyceride clearance in liver," Endocrinology, vol. 150, no. 10, pp. 4625-4633, 2009.

[33] K. Mai, J. Andres, K. Biedasek et al., "Free fatty acids link metabolism and regulation of the insulin-sensitizing fibroblast growth factor-21," Diabetes, vol. 58, no. 7, pp. 1532-1538, 2009.

[34] D. D. Moore, "Sister act," Science, vol. 316, no. 5830, pp. 1436 1438, 2007.

[35] M. Kuro-o, "Endocrine FGFs and Klothos: emerging concepts," Trends in Endocrinology and Metabolism, vol. 19, no. 7, pp. 239-245, 2008.

[36] M. D. L. Chau, J. Gao, Q. Yang, Z. Wu, and J. Gromada, "Fibroblast growth factor 21 regulates energy metabolism by activating the AMPK-SIRT1-PGC- $1 \alpha$ pathway," Proceedings of the National Academy of Sciences of the United States of America, vol. 107, no. 28, pp. 12553-12558, 2010.

[37] S. Kersten, J. Seydoux, J. M. Peters, F. J. Gonzalez, B. Desvergne, and W. Wahli, "Peroxisome proliferator-activated receptor $\alpha$ mediates the adaptive response to fasting," Journal of Clinical Investigation, vol. 103, no. 11, pp. 1489-1498, 1999.

[38] T. C. Leone, C. J. Weinheimer, and D. P. Kelly, "A critical role for the peroxisome proliferator-activated receptor $\alpha(\operatorname{PPAR} \alpha)$ in the cellular fasting response: the PPAR $\alpha$-null mouse as a model of fatty acid oxidation disorders," Proceedings of the National Academy of Sciences of the United States of America, vol. 96, no. 13, pp. 7473-7478, 1999.

[39] C. Gälman, T. Lundåsen, A. Kharitonenkov et al., "The circulating metabolic regulator FGF21 is induced by prolonged fasting and PPARalpha activation in man," Cell Metabolism, vol. 8, no. 2, pp. 169-174, 2008.

[40] C. Christodoulides, P. Dyson, D. Sprecher, K. Tsintzas, and F. Karpe, "Circulating fibroblast growth factor 21 is induced by peroxisome proliferator-activated receptor agonists but not ketosis in man," Journal of Clinical Endocrinology and Metabolism, vol. 94, no. 9, pp. 3594-3601, 2009.

[41] M. K. Badman, A. Koester, J. S. Flier, A. Kharitonenkov, and E. Maratos-Flier, "Fibroblast growth factor 21-deficient mice demonstrate impaired adaptation to ketosis," Endocrinology, vol. 150, no. 11, pp. 4931-4940, 2009.
[42] J. L. Estall, J. L. Ruas, C. S. Choi et al., "PGC- $1 \alpha$ negatively regulates hepatic FGF21 expression by modulating the heme/Rev$\operatorname{Erb} \alpha$ axis," Proceedings of the National Academy of Sciences of the United States of America, vol. 106, no. 52, pp. 22510-22515, 2009.

[43] M. J. Potthoff, T. Inagaki, S. Satapati et al., "FGF21 induces PGC- $1 \alpha$ and regulates carbohydrate and fatty acid metabolism during the adaptive starvation response," Proceedings of the National Academy of Sciences of the United States of America, vol. 106, no. 26, pp. 10853-10858, 2009.

[44] J. P. Thissen, L. E. Underwood, and J. M. Ketelslegers, "Regulation of insulin-like growth factor-I in starvation and injury," Nutrition Reviews, vol. 57, no. 6, pp. 167-176, 1999.

[45] T. Inagaki, V. Y. Lin, R. Goetz, M. Mohammadi, D. J. Mangelsdorf, and S. A. Kliewer, "Inhibition of growth hormone signaling by the fasting-induced hormone FGF21," Cell Metabolism, vol. 8, no. 1, pp. 77-83, 2008.

[46] R. G. Melvin and M. T. Andrews, "Torpor induction in mammals: recent discoveries fueling new ideas," Trends in Endocrinology and Metabolism, vol. 20, no. 10, pp. 490-498, 2009.

[47] S. Chikahisa, K. Tominaga, T. Kawai et al., "Bezafibrate, a peroxisome proliferator-activated receptors agonist, decreases body temperature and enhances electroencephalogram deltaoscillation during sleep in mice," Endocrinology, vol. 149, no. 10, pp. 5262-5271, 2008.

[48] K. Oishi, K. Sakamoto, M. Konishi, Y. Murata, N. Itoh, and H. Sei, "FGF21 is dispensable for hypothermia induced by fasting in mice," Neuroendocrinology Letters, vol. 31, no. 2, pp. 198202, 2010.

[49] N. Turner and R. Grose, "Fibroblast growth factor signalling: from development to cancer," Nature Reviews Cancer, vol. 10, no. 2, pp. 116-129, 2010.

[50] M. Kuro-o, Y. Matsumura, H. Aizawa et al., "Mutation of the mouse klotho gene leads to a syndrome resembling ageing," Nature, vol. 390, no. 6655, pp. 45-51, 1997.

[51] S. Ito, S. Kinoshita, N. Shiraishi et al., "Molecular cloning and expression analyses of mouse $\beta$ klotho, which encodes a novel Klotho family protein," Mechanisms of Development, vol. 98, no. 1-2, pp. 115-119, 2000.

[52] I. Urakawa, Y. Yamazaki, T. Shimada et al., "Klotho converts canonical FGF receptor into a specific receptor for FGF23," Nature, vol. 444, no. 7120, pp. 770-774, 2006.

[53] S. Ito, T. Fujimori, A. Furuya, J. Satoh, Y. Nabeshima, and Y. I. Nabeshima, "Impaired negative feedback suppression of bile acid synthesis in mice lacking $\beta$ Klotho," Journal of Clinical Investigation, vol. 115, no. 8, pp. 2202-2208, 2005.

[54] C. Yu, F. Wang, M. Kan et al., "Elevated cholesterol metabolism and bile acid synthesis in mice lacking membrane tyrosine kinase receptor FGFR4," Journal of Biological Chemistry, vol. 275, no. 20, pp. 15482-15489, 2000.

[55] T. Inagaki, M. Choi, A. Moschetta et al., "Fibroblast growth factor 15 functions as an enterohepatic signal to regulate bile acid homeostasis," Cell Metabolism, vol. 2, no. 4, pp. 217-225, 2005.

[56] H. Kurosu, M. Choi, Y. Ogawa et al., "Tissue-specific expression of $\beta$ klotho and Fibroblast Growth Factor (FGF) receptor isoforms determines metabolic activity of FGF19 and FGF21," Journal of Biological Chemistry, vol. 282, no. 37, pp. 2668726695, 2007.

[57] Y. Ogawa, H. Kurosu, M. Yamamoto et al., " $\beta$ Klotho is required for metabolic activity of fibroblast growth factor 21," Proceedings of the National Academy of Sciences of the United States of America, vol. 104, no. 18, pp. 7432-7437, 2007. 
[58] A. Kharitonenkov, J. D. Dunbar, H. A. Bina et al., "FGF21/FGF-21 receptor interaction and activation is determined by $\beta$ Klotho," Journal of Cellular Physiology, vol. 215, no. 1, pp. $1-7,2008$.

[59] M. Suzuki, Y. Uehara, K. Motomura-Matsuzaka et al., " $\beta$ klotho is required for fibroblast growth factor (FGF) 21 signaling through FGF receptor (FGFR) 1c and FGFR3c," Molecular Endocrinology, vol. 22, no. 4, pp. 1006-1014, 2008.

[60] K. I. Tomiyama, R. Maeda, I. Urakawa et al., "Relevant use of Klotho in FGF19 subfamily signaling system in vivo," Proceedings of the National Academy of Sciences of the United States of America, vol. 107, no. 4, pp. 1666-1671, 2010.

[61] G. Morris-Stiff and A. E. Feldstein, "Fibroblast growth factor 21 as a biomarker for NAFLD: integrating pathobiology into clinical practice," Journal of Hepatology, vol. 53, no. 5, pp. 795796, 2010.

[62] E. Bugianesi, S. Moscatiello, M. F. Ciaravella, and G. Marchesini, "Insulin resistance in nonalcoholic fatty liver disease," Current Pharmaceutical Design, vol. 16, no. 17, pp. 1941-1951, 2010.

[63] Y. Yilmaz, F. Eren, O. Yonal et al., "Increased serum FGF21 levels in patients with nonalcoholic fatty liver disease," European Journal of Clinical Investigation, vol. 40, no. 10, pp. 887-892, 2010.

[64] J. Dushay, P. C. Chui, G. S. Gopalakrishnan et al., "Increased fibroblast growth factor 21 in obesity and nonalcoholic fatty liver disease," Gastroenterology, vol. 139, no. 2, pp. 456-463, 2010.

[65] H. Li, Q. Fang, F. Gao et al., "Fibroblast growth factor 21 levels are increased in nonalcoholic fatty liver disease patients and are correlated with hepatic triglyceride," Journal of Hepatology, vol. 53, no. 5, pp. 934-940, 2010.

[66] W. W. Chen, L. Li, G. Y. Yang et al., "Circulating FGF-21 levels in normal subjects and in newly diagnose patients with type 2 diabetes mellitus," Experimental and Clinical Endocrinology and Diabetes, vol. 116, no. 1, pp. 65-68, 2008.

[67] X. Zhang, D. C. Y. Yeung, M. Karpisek et al., "Serum FGF21 levels are increased in obesity and are independently associated with the metabolic syndrome in humans," Diabetes, vol. 57, no. 5, pp. 1246-1253, 2008.

[68] A. O. Chavez, M. Molina-Carrion, M. A. Abdul-Ghani, F. Folli, R. A. DeFronzo, and D. Tripathy, "Circulating fibroblast growth factor-21 is elevated in impaired glucose tolerance and type 2 diabetes and correlates with muscle and hepatic insulin resistance," Diabetes Care, vol. 32, no. 8, pp. 1542-1546, 2009.

[69] M. Mraz, M. Bartlova, Z. Lacinova et al., "Serum concentrations and tissue expression of a novel endocrine regulator fibroblast growth factor-21 in patients with type 2 diabetes and obesity," Clinical Endocrinology, vol. 71, no. 3, pp. 369375, 2009.

[70] S. Stein, H. Stepan, J. Kratzsch et al., "Serum fibroblast growth factor 21 levels in gestational diabetes mellitus in relation to insulin resistance and dyslipidemia," Metabolism, vol. 59, no. 1, pp. 33-37, 2010.

[71] B. Matuszek, M. Lenart-Lipińska, D. Duma, J. Solski, and A. Nowakowski, "Evaluation of concentrations of FGF-21-a new adipocytokine in type 2 diabetes," Endokrynologia Polska, vol. 61 , no. 1 , pp. 50-54, 2010.

[72] F. M. Fisher, P. C. Chui, P. J. Antonellis et al., "Obesity is a fibroblast growth factor 21 (FGF21)-resistant state," Diabetes, vol. 59, no. 11, pp. 2781-2789, 2010.

[73] H. Li, Y. Bao, A. Xu et al., "Serum fibroblast growth factor 21 is associated with adverse lipid profiles and $\gamma$-glutamyltransferase but not insulin sensitivity in Chinese subjects,"
Journal of Clinical Endocrinology and Metabolism, vol. 94, no. 6, pp. 2151-2156, 2009.

[74] V. Durovcová, J. Marek, V. Hána et al., "Plasma concentrations of fibroblast growth factors 21 and 19 in patients with Cushing's syndrome," Physiological Research, vol. 59, no. 3, pp. 415-422, 2010.

[75] F. Villarroya, P. Domingo, and M. Giralt, "Lipodystrophy in HIV 1-infected patients: lessons for obesity research," International Journal of Obesity, vol. 31, no. 12, pp. 1763-1776, 2007.

[76] P. Domingo, J. M. Gallego-Escuredo, J. C. Domingo et al., "Serum FGF21 levels are elevated in association with lipodystrophy, insulin resistance and biomarkers of liver injury in HIV-1-infected patients," AIDS, vol. 24, no. 17, pp. 26292637, 2010.

[77] S. Stei, A. Bachmann, U. Lössner et al., "Serum levels of the adipokine FGF21 depend on renal function," Diabetes Care, vol. 32, no. 1, pp. 126-128, 2009.

[78] S. H. Han, S. H. Choi, B. J. Cho et al., "Serum fibroblast growth factor-21 concentration is associated with residual renal function and insulin resistance in end-stage renal disease patients receiving long-term peritoneal dialysis," Metabolism, vol. 59, no. 11, pp. 1656-1562, 2010. 


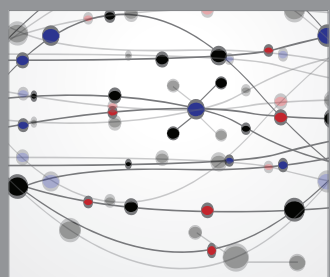

The Scientific World Journal
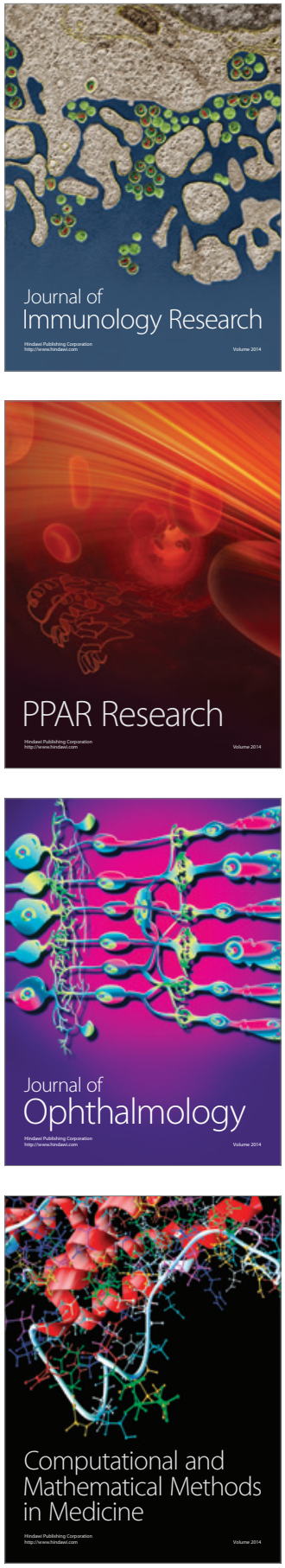

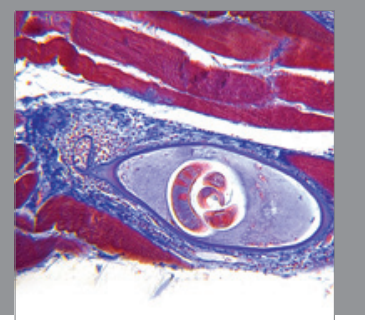

Gastroenterology

Research and Practice
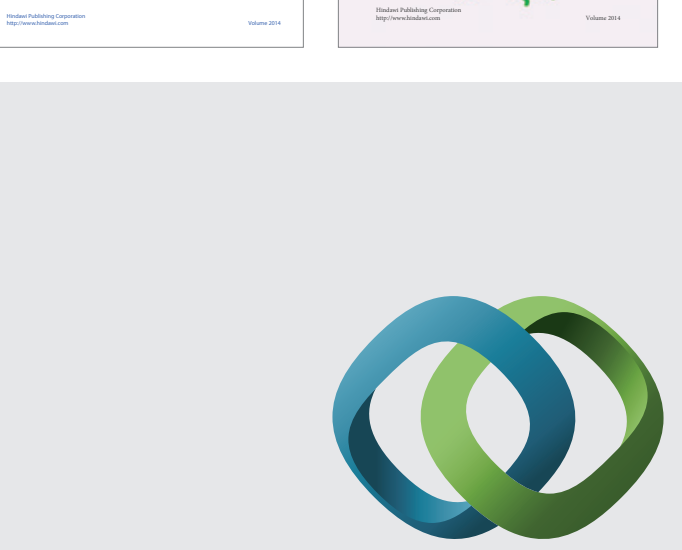

\section{Hindawi}

Submit your manuscripts at

http://www.hindawi.com
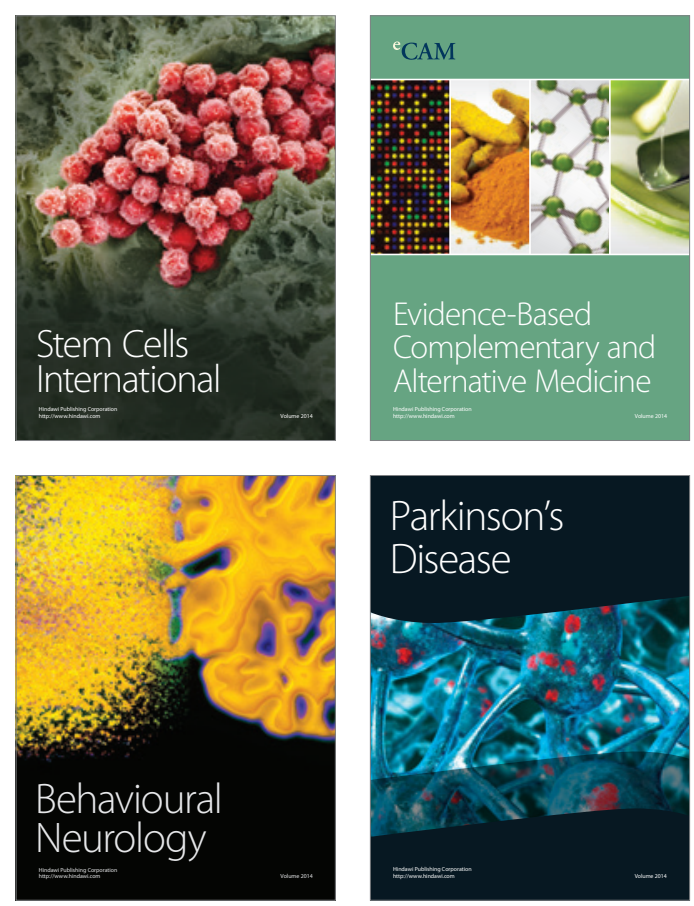

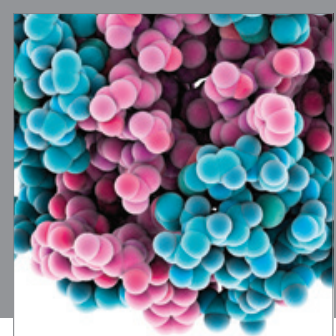

Journal of
Diabetes Research

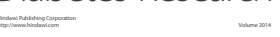

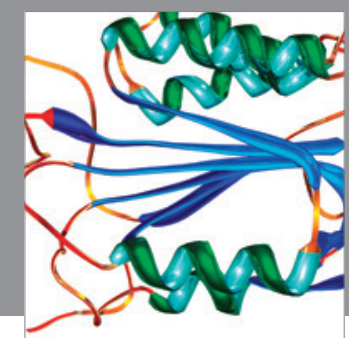

Disease Markers
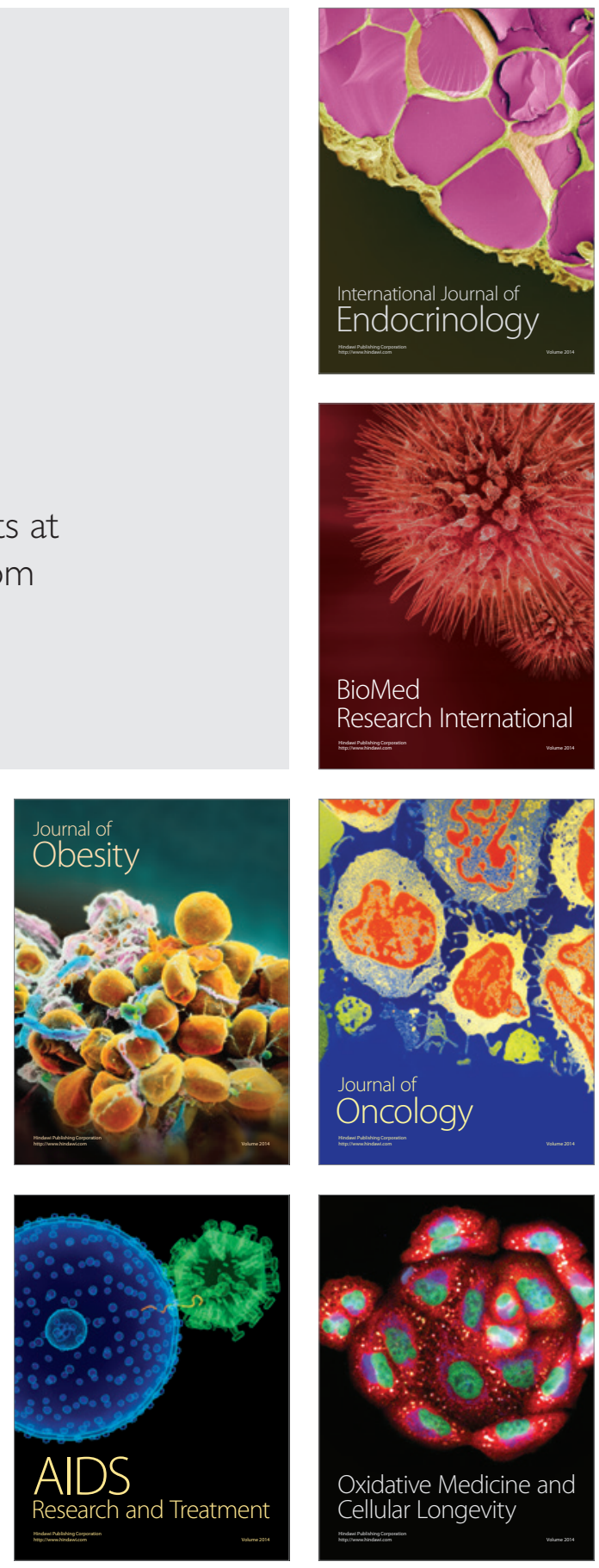